\title{
Analysis of the Heart Rate Variability in Negative Functional States in the Course of Psychological Relaxation Sessions
}

\author{
V. A. Mashin and M. N. Mashina \\ Novovoronezh Center for Training Nuclear Power Station (NPS) Specialists, Novovoronezh, Russia \\ Received August 3, 1998
}

\begin{abstract}
The aim of this study was to compare the effects of psychological relaxation on the heart rate variability (HRV) in subjects with a number of negative functional states: asthenia, depression, and neurotic symptoms. The analysis performed allowed us to reveal the dynamics of the $H R V$ variables characteristic of each negative functional state and to determine indicators, which are significant for their diagnosis. The effectiveness of using $H R V$ analysis for the control and management of the psychological relaxation process and the diagnosis of a number of negative functional states in humans is substantiated by the study results.
\end{abstract}

In 1997, a special course for the development of self-regulation and autotraining habits in NPS operators that included the system of psychotechnical exercises, autogenic immersion according to M.M. Reshetnikov's variant, and psychological relaxation sessions based on the method of image identification using theatrical and musical compositions was developed at the Psychophysiological Survey Laboratory (PSL) of the Novovoronezh Center for Training NPS Personnel. Physiological control of the functional state of the human during psychological relaxation played an important role when administering this course. To solve this task, we decided to use the heart rate variability (HRV) analysis that is used at the PSL when carrying out a psychophysiological survey of NPS personnel. HRV analysis gained wide acceptance over the last ten years as a method that allows us to quantitatively characterize the activity of different divisions of the autonomic nervous system (ANS) through their influence on the function of the sinus node of the heart, which is reflected in variations in the duration of the RR-intervals [I]. We decided to follow the change in $H R V$ in the process of psychological relaxation for groups of operators with different negative states: neurosis, depression, and depletion of functional reserves (asthenia).

\section{METHODS}

Fifty-six subjects (mean age 31.2 years, standard deviation 6.1), who underwent an in-depth psycho-physiological survey at the PSL, participated in the study. Based on the survey results, four groups were formed: norm $(n=18)$; asthenic state $(n=$ 11); depressive state $(n=12)$; neurotic state $(n=15)$. All the groups were age-matched. Psychological relaxation sessions were conducted in the second half of the instruction period. In the course of self-regulation sessions, the physiological state of the subjects was controlled by recording cardiac rhythm $R R$-intervals using the Grand complex with subsequent $H R V$ analysis. Temporal and spectral $H R V$ analysis and the following parameters were used in this study [2]: $r M S S D(\mathrm{~ms})$ is the standard deviation of differences between the duration of adjacent sinus $R R$-intervals, it reflects the activity of the parasympathetic division of the ANS (PNS); $p N N_{50}(\%)$ is the fraction of adjacent sinus $R R$-intervals, which differ more than $50 \mathrm{ms,} \mathrm{it} \mathrm{reflects}$ the activity of the parasympathetic division of the ANS; SDNN (ms) is the standard deviation from the average duration of all sinus $R R$-intervals, it is the integral parameter of the overall activity of regulatory systems; $P W$ (PoWer spectrum, beats $/ \mathrm{min}^{2}$ ) is the overall power of the cardiac rhythm spectrum, it reflects the overall activity of regulatory systems; VLF (beats $/ \mathrm{min}^{2}$ ) is the power in a very low-frequency $0.00-0.04-\mathrm{Hz}$ range, the physiological nature is not clear; $L F$ (beats $/ \mathrm{min}^{2}$ ) is the power in a low-frequency $0.04-0.15-\mathrm{Hz}$ range, it reflects, mainly, the activity of the sympathetic division of the ANS (SNS); HF $\left(\right.$ beats $/ \mathrm{min}^{2}$ ) denotes the power in the high-frequency $0.15-0.40-\mathrm{Hz}$ range, it indicates the activity of the parasympathetic division of the ANS; LFIHF reflects the balance of the sympathetic and parasympathetic divisions of the ANS; and (LF + $H F) / P W(\%)$ is the share of the summary power of the parasympathetic and sympathetic divisions of the ANS in the total spectrum power $(n L H)$. Additionally, $r M S S D$ and $S D N N$ values were calculated by the pulse rate ( $r M S S D a, S D N N a$, beats $/ \mathrm{min}$ ). Artifacts (extra beats, interference) were visually removed from the initial recordings and selected for subsequent processing.

Taking into account the specificity of the psycho-physiological surveys, we determined the standard sample equal to 256 $R R$-intervals $[3,4]$. To analyze the dynamics of the process, processing was carried out with a sliding sample with a step of 10 $R R$-intervals. Spectral analysis of beat-to-beat rate data was carried out using the fast Fourier transform algorithm [5-7] and Hamming's spectral window. The stability of the samples obtained was determined with Spearman's rank coefficient of correlation for the mean and the variance separately [8]. If the sample met the requirement of stability for both parameters, it was included for subsequent spectral analysis. After discarding the recordings inadequate for processing, editing the artifacts, and checking for stability, 5949 samples with $256 R R$-intervals were selected for subsequent analysis. The calculation and removal of the trend for spectral analysis was carried out using the least squares analysis. Standard deviations and variation coefficients were calculated for all the variables based on the sliding sample data.

Two types of psychological relaxation sessions were used in the study, namely, autogenic immersion according to M.M. Reshetnikov's variant and theatrical and musical compositions to achieve the relaxation effect ("Rain," "The Flow of Consciousness"), in which the elements of autogenic training were combined with deep immersion of consciousness into the flow of verbal and musical images. The duration of sessions was about 20 to $30 \mathrm{~min}$. Each session was preceded by exercises for general tranquilization, concentration of attention, and relaxation technique. 
Three cardiac rhythm samples were recorded: (1) before a session (of about 5-min duration), (2) during a session (of 20- to 30-min duration), and (3) after a session (of about 5-min duration). In the process of recording, a subject was in a quiet state in an armchair with a high back, the arms lying on the armrests.

\section{RESULTS AND DISCUSSION}

Preliminarily, we analyzed with correlation analysis the interrelationship between temporal and spectral $H R V$ variables. For this purpose, we used the data obtained during psychological relaxation sessions (the number of samples is 3740 ).

\begin{tabular}{|c|c|c|c|c|c|c|c|c|c|c|}
\hline & Pulse rate & $R R$ & $P W$ & $V L F$ & $L F$ & $H F$ & $n L H$ & LFIHF & rMSSDa & SDNNa \\
\hline$S D N N$ & -0.49 & 0.50 & 0.80 & 0.44 & 0.72 & 0.76 & 0.12 & 0.13 & 0.82 & 0.80 \\
\hline rMSSD & -0.61 & 0.61 & 0.58 & 0.13 & 0.54 & 0.81 & 0.36 & -0.17 & 0.85 & 0.57 \\
\hline$p N N_{50}$ & -0.65 & 0.64 & 0.42 & 0.05 & 0.36 & 0.69 & 0.38 & -0.22 & 0.70 & 0.44 \\
\hline SDNNa & 0.08 & -0.08 & 0.96 & 0.74 & 0.81 & 0.72 & -0.00 & 0.30 & 0.76 & - \\
\hline rMSSDa & -0.20 & 0.18 & 0.81 & 0.30 & 0.75 & 0.96 & 0.32 & -0.06 & - & \\
\hline
\end{tabular}

Table 1. Spearman's correlation coefficients for temporal and spectral $H R V$ variables

According to the results obtained (Table 1), all temporal parameters calculated by $R R$-intervals $\left(S D N N, r M S S D\right.$, and $\left.p N N_{50}\right)$ have a very high correlation both with the pulse rate $(\mathrm{PR})$ and $R R$ duration (intercorrelation between $\mathrm{PR}$ and $R R$ was $-0.98, p=$ $0.00)$. In turn, the pulse rate calculated temporal analysis parameters have a weak correlation with these variables (this is especially true of SDNNa). The comparison of the SDNN and SDNNa coefficients of correlation with the pulse rate (-0.49 and $0.08)$ and $R R$-intervals $(0.50$ and -0.08$)$ points to their significant differences (z-statistics is equal to 58.46 for the pulse rate and 59.46 for $R R$-intervals, $p=0.00)$. Similar differences between the coefficients of correlation and PR and $R R$ were obtained for $r M S S D$ and $r M S S D a$ variables (z-statistics is equal to 45.78 and 47.68 , respectively, $p=0.00$ ). Since both $r M S S D$ and $H F$ reflect the activity of the parasympathetic nervous system, we compared the rMSSD and rMSSDa coefficients of correlation with $H F$. It was established that the standard deviation of differences between the duration of the adjacent sinus $R R$-intervals has a statistically lower coefficient of correlation with the power of waves in the HF range than this parameter calculated by the pulse rate (z-statistics is equal to $29.69, p=0.00)$. It is known that spectral analysis allows the variance of the studied temporal series to be resolved by the frequency range. It follows that the total spectrum power ( $P W)$ should have a high correlation with the standard deviation $(S D N N)$. The comparison of the coefficients of correlation of the temporal SDNN and SDNNa values with $P W$ allowed a conclusion about a higher correlation between SDNNa and PW (z-statistics for the two coefficients of correlation is $29.31, p=0.00)$ to be made. Analysis of the SDNNa versus $P W$ plot revealed the exponential dependence of these parameters. Regression analysis yielded the following formula: $S D N N a=-0.209+3.554 \cdot P W^{0.487}(R=$ 0.992, $p=0.00)$.

The results of the correlation analysis confirmed the temporal $H R V$ values calculated by the pulse rate conclusively enough. That is why we abandoned $S D N N$, rMSSD, and $p N N_{50}$ in our further analysis values and focused on the spectral analysis data and the temporal SDNNa and rMSSDa values.

Analysis of the dynamics of the normalized parameter of the summary power of the parasympathetic and sympathetic divisions of the ANS $(n L H)$ permitted three groups of subjects to be singled out. The first group included individuals who had a stable $n L H$ increase (above 60 to $70 \%$ ). The subjects with $n L H$ varying in the $40-60 \%$ range were included in the second group; the third group included those who had stable low values of this parameter (less than $40 \%$ ). Note that a decrease or an increase in the share of the $V L F$ range wave power in the total spectrum corresponds to an increase or a decrease in the $n L H$ values.

At present, it is unclear what reflects the spectrum power in the VLF range. According to the neurophysiological data, autonomic "centers" influencing the activity of the cardiovascular system through the peripheral divisions of the ANS are situated in the medulla oblongata. These autonomic centers are, in turn, controlled by the hypothalamus, which is, in vertebrates, the main center responsible for regulating the internal environment of the body and exercising control over all the main homeostatic processes. Under emotional stress, the ANS activity is affected by the limbic system and the cortical structures of the brain. These divisions influence the cardiovascular system through the hypothalamus and midbrain as well as the spinal cord. According to many investigators, the values of the spectrum power in the low- and high-frequency ranges reflect the activity of the sympathetic and parasympathetic divisions of the ANS (the autonomic centers of the medulla oblongata and hypothalamus). We suggested that the spectrum power in the VLF range was indicative of the predominant influence of the corticolimbic divisions of the brain on the regulation of cardiac rhythm. This supposition is in keeping with the hypothesis of the two-circuit system of cardiac rhythm control advanced by R.M. Baevskii as far back as the mid-1970s, which includes the higher autonomic centers being controlled by cortical mechanisms that coordinate all the control processes of the body in accordance with environmental conditions $[9,10]$. According to this hypothesis, the lower the frequency of the heart rhythm variations, the higher the level of control. Nonoptimal control requires activation of higher levels of regulation. This is manifested by an increase in the nonrespiratory components of sinus arrhythmia, by the appearance of slow waves with 
increasingly higher periods, and by an increase in their power.

The results of our investigations were of crucial importance for formulating an hypothesis about the corticolimbic nature of power in the VLF range. All the subjects from the group with an increased spectrum power in the VLF range appeared to be in a state of intense emotional stress or to have marked neurotic symptoms at the time of psychological relaxation sessions. We cite three examples (Table 2).

\begin{tabular}{l|l|l|l|l|l|l|l}
\hline Subject, test no. & Pulse rate & $P W$ & VLF & LF & HF & LFIHF & $n L H$ \\
\hline Sh. 1 & $87.12(2.30)$ & $17.41(3.54)$ & $7.39(1.41)$ & $7.83(1.61)$ & $2.33(0.51)$ & $3.51(0.67)$ & $59 \%(10)$ \\
Sh. 2 & $83.44(2.37)$ & $28.78(7.11)$ & $20.53(4.36)$ & $5.91(1.54)$ & $2.22(0.76)$ & $2.88(1.14)$ & $29 \%(7)$ \\
Sh. 3 & $78.04(2.28)$ & $19.72(5.50)$ & $9.64(1.45)$ & $6.45(1.13)$ & $3.64(0.59)$ & $1.75(0.23)$ & $53 \%(8)$ \\
B. 1 & $72.48(0.23)$ & $15.81(1.71)$ & $8.54(0.55)$ & $6.24(0.66)$ & $1.19(0.14)$ & $5.33(0.77)$ & $51 \%(5)$ \\
B. 2 & $70.37(1.01)$ & $6.39(0.74)$ & $4.71(1.03)$ & $1.40(0.37)$ & $0.28(0.10)$ & $4.51(1.21)$ & $27 \%(5)$ \\
B. 3 & $72.76(1.78)$ & $20.63(0.70)$ & $9.06(0.93)$ & $10.31(1.22)$ & $1.21(0.13)$ & $9.13(1.55)$ & $56 \%(6)$ \\
K. 1 & $68.62(1.10)$ & $16.21(4.30)$ & $5.87(1.34)$ & $6.03(1.92)$ & $4.41(0.54)$ & $1.31(0.30)$ & $65 \%(8)$ \\
K. 2 & $65.34(1.04)$ & $15.49(1.33)$ & $3.45(0.30)$ & $5.28(1.34)$ & $6.87(0.45)$ & $0.78(0.24)$ & $78 \%(3)$ \\
K. 3 & $74.38(1.63)$ & $28.43(1.50)$ & $15.81(0.92)$ & $7.81(0.53)$ & $4.79(0.41)$ & $1.61(0.22)$ & $45 \%(2)$ \\
\hline
\end{tabular}

Table 2. Means and standard deviations fin brackets) for the main $H R V$ values of subjects Sh., B. and K. for each test

Before a session, subject Sh. experienced a severe emotional stress. During the session, he was evidently overexcited. While an increased pulse rate, lability of the $H R V$ values (the coefficient of variability is more than $20 \%$ ), as well as the predominance of the activity of the sympathetic nervous system $\{L F I H F=3.51)$ are noted at rest before the session, during the psychological relaxation session, a sharp increase in the wave power in the VLF range (from 7.39 to 20.53) and an $n L H$ decrease (from 59 to 29) were observed. If we follow the supposition that VLF and the activity of the corticolimbic structures (CLS) are interrelated, not only did the influence of an emotionally experienced event not decrease during the session, but it increased still more. This is proven by the subject's self-account data, according to which not only could he not relax during the session, but, on the contrary, his emotional excitation increased as a result of his mentally experiencing what had happened. Possibly, due to this irrational hyperactivity of the corticolimbic structures, the enhancement of neurotic symptoms wellknown to clinicians occurs when an individual is at physical rest. It is significant that after the session, the mobilization phase usually observed in this operator was absent. On the contrary, the pulse rate was increasingly decreased, the VLF and $n L H$ values approached the initial values, and the autonomic balance shifted toward parasympathetic influences. The positive effect of psychological relaxation showed up immediately after the session. Later, this positive effect was observed during the session, and, by the end of the course, the subject's condition almost normalized.

The following example concerns subject B. who had marked neurotic symptoms for a long time. As in the first case, a distinct $n L H$ decrease was characteristic of the second test. The $L F I H F$ value indicates the predominance of sympathetic influences in all the tests. During the session, the total spectrum power $(P W)$ decreased almost by $60 \%$ (the power dramatically decreased in the LF- and //F-ranges). In his self-account, the subject pointed out that "his muscles, but not his conscience, relaxed."

Quite a different picture was observed in the normal group, for which increased $n L H$ values were characteristic during the session. Table 2 shows the data of subject $\mathrm{K}$. who participated in the psychological self-regulation session a second time. Good relaxation was noted before the session. This was evidenced by a decrease in the autonomic balance value $(L F / L H)$. During the session, as judged by the self-account data, the subject achieved deep relaxation and fell asleep. It is important for us that this was accompanied not only by an increase in the activity of the parasympathetic division of the ANS, but also by high $n L H$ values, which, according to our supposition, might be evidence in favor of the predominant influence of the ANS in the regulation of cardiac rhythm. One-factor analysis confirmed a significant $n L H$ decrease in neurotization $(p=0.00)$.

Important information was obtained from analyzing the $n L H$ histogram. The histogram of the second test results showed two partially intersecting (approximately at point 48), but distinctly discernible distributions. The first distribution $(n L H<48)$ had the average value equal to 35.70, standard deviation 6.89; the second distribution ( $n L H \geq 48$ ), 70.8 and 10.2, respectively. The data obtained allowed us to specify the $n L H$ boundaries of the groups: the first group, the predominance of the CLS influence, $n L H<42.7$; the second group, the balance between the CLS and ANS $(42.7 \leq n L H<60.6)$; the third group, the predominant influence of the ANS $(n L H \geq 60.6)$.

Having grouped the subjects by the $n L H$ values (according to our data, they reflect the level of centralization of the regulation of cardiovascular activity), we proceeded to classify the subjects by autonomic balance $(V B=L F / H F)$ and total spectrum power $(P W)$ values during the psychological relaxation sessions. Three levels of autonomic balance were singled out: $V B<0.95\left(\mathrm{VB}_{1}\right)$, the predominance of the PNS influence; $0.95 \leq V B<1.88\left(\mathrm{VB}_{2}\right)$, the balance between the PNS and SNS; and $V B \geq 1.88\left(\mathrm{VB}_{3}\right)$, the predominance of the SNS.

Three levels were also singled out based on the total spectrum power values (the fourth level with very high power values above 25 constituted an insignificant group whose physiological nature requires further investigation): $P W<4.2$, a low-power 
spectrum; $4.2 \leq P W<13.5$, average values; and $P W \geq 13.5$, a high-power spectrum.

Such a classification allowed us to establish that, in the course of psychological relaxation sessions, a decrease in the total spectrum power to low values is characteristic of the group with decreased functional reserves (Table 3 ).

\begin{tabular}{|c|c|c|c|c|c|c|c|c|c|}
\hline Group & Pulse rate & $P W$ & $V L F$ & $L F$ & $H F$ & $L F / H F$ & $n L H$ & SDNNa & rMSSDa \\
\hline \multirow[t]{2}{*}{1} & 67.97 & 11.53 & 2.90 & 3.34 & 5.29 & .63 & 75.10 & 3.42 & 11.53 \\
\hline & (11.38) & (9.53) & $(2.86)$ & (3.02) & (4.34) & $(.25)$ & (10.41) & (1.32) & (9.98) \\
\hline \multirow[t]{2}{*}{2} & 72.39 & 3.05 & 1.02 & 1.25 & .78 & 1.71 & 65.92 & 1.84 & 2.34 \\
\hline & $(9.80)$ & (.63) & (.33) & $(.37)$ & $(.25)$ & (.49) & $(8.86)$ & $(.22)$ & $(.78)$ \\
\hline \multirow[t]{2}{*}{3} & 69.04 & 2.70 & .82 & .62 & 1.26 & .54 & 69.90 & 1.78 & 3.40 \\
\hline & $(4.73)$ & $(.38)$ & $(.28)$ & $(.12)$ & $(.29)$ & $(.15)$ & (7.39) & $(.15)$ & $(.76)$ \\
\hline \multirow[t]{2}{*}{4} & 73.99 & 14.91 & 10.10 & 3.26 & 1.55 & 2.72 & 32.66 & 3.93 & 4.34 \\
\hline & $(9.29)$ & (9.79) & (6.93) & (2.09) & (1.17) & (1.47) & $(5.71)$ & (1.41) & (2.79) \\
\hline \multirow[t]{2}{*}{ Mean } & 70.35 & 9.65 & 3.95 & 2.60 & 3.09 & 1.31 & 62.96 & 3.04 & 7.16 \\
\hline & $(9.12)$ & (8.05) & (4.99) & (2.07) & (2.98) & (1.16) & (18.68) & $(1.26)$ & $(6.46)$ \\
\hline
\end{tabular}

Table 3. Means and standard deviations (in brackets) of the main $H R V$ values during psychological relaxation for different groups of subjects (1, normal, 2, asthenia, 3, depressive states, 4, neurotic states)

The comparison of means for the normal and asthenia groups using Student's test gave a significant decrease in the $H R V$ values in the second group $(t=16.85$ to $30.41 ; p=0.00)$. According to one-factor analysis, the share of variation of the total spectrum power was $44.54 \%$. The fact of a decrease in heart rate variability with age reported in a number of investigations $[11,12]$ is likely due to the overall decrease in the functional reserves of the human body.

In the group of subjects in whom depression was diagnosed on the basis of the results of psychological testing (including $M M P I$ and a clinical interview), along with the total power decrease (which unites them with the second group), significantly lower $L F / H F$ ratio values $(t=5.87 ; p=0.00)$ were noted, which indicated the high prevalence of the PNS in the total autonomic balance (although the absolute values of both $H F$ and $r M S S D a$ are much higher in the first group). High $n L H$ values are characteristic of all the first three groups. The data obtained are confirmed by the results of the study of depressive states by foreign scientists. In one of them [13], in the course of treating depressive patients, a statistically significant relationship between the improvement of the patients' state according to Hamilton's depression scale and a decrease in the spectrum power in the HF-range (and, correspondingly, an increase in the LF/HF ratio) was found. In the other study [14], a review of the means of management of depressive states that accompany such diseases as coronary artery disorders, cancer, and the immune deficiency syndrome was made. It was established that severe depression significantly increases the mortality rate in patients with coronary artery disorders. The authors emphasize that the HRV decrease is a distinctive feature of depressive patients. The depressive state typical of severely ill patients might be the body's response to chronic stress, which manifests itself by a passive refusal to struggle. This refusal reflects both the state of the body (the absence of the functional reserve to resist the internal sources of disease) and the subjective state of the human being (the patient's assessment of a situation). This passive reaction may contribute to a decrease in the total spectrum power and the predominance of the PNS in the autonomic balance. It is significant that low $P W$ values (less than $2.5 \%$ of all samples for a given group had a spectrum power below 4.2 during the session) are not characteristic of neurotic states. That neurotization is an active form of the body's reaction (consciousness) to the existing discrepancy, while depression is a passive form of this reaction, may be a possible explanation.

The comparison of the results of the heart rate variability in the process of psychological relaxation for the normal and neurotic groups reveals a significant decrease in the $n L H$ value $(L F+H F)$ norm, to which an increase in the spectrum power in the VLF-range ( $t=99.24$ and -26.65 , respectively; $p=0.00)$ corresponds. The fourth group is also characterized by a decrease in the HF and $r M S S D a$ values, an increase in the $L F / H F$ ratio (a low PNS activity, $t=23.11,19.26$, and $-38,79$, respectively; $p$ $=0.00$ ), and virtually the absence of samples with a low total spectrum power. According to our supposition, a decrease in the $n L H$ value during a psychological relaxation session may testify to the predominant activity of corticolimbic structures in regulating the cardiac rhythm. It follows from our results that the activity of the ANS mainly decreases due to the PNS.

According to the spectral analysis data of $H R V$, one could have a clear idea of the effect of psychological relaxation sessions on specific subjects. Earlier, we gave an example of the positive dynamics of an extremely emotional state of one of the subjects (Sh.) in the course of the sessions. Note that, on the first recording of cardiac rhythm in the subject, $100 \%$ of the samples were characterized by high values in terms of CLS; 77\% in terms of SNS (0\% PNS); and $100 \%$ by high total spectrum power values ( $80 \%$ of them are very high). During the second recording, this subject showed $48 \%$ ANS (0\% CLS), $82 \%$ PNS ( $0 \%$ SNS), $62 \%$ of the average power and $38 \%$ of high power.

We shall give another example of the positive dynamics of the $H R V$ values during psychological relaxation sessions. The first recording revealed the following results: CLS, 37\% (ANS 13\%); SNS, 91\% (PNS 0\%); average power, 100\%. The selfaccount stated that "no relaxation was achieved." During the second recording the results were as follows: ANS, 75\% (CLS $0 \%$ ); SNS, 3\% (PNS 4\%); $P W_{1}, 57 \% ; P W_{2}, 43 \%$. According to the self-account, the subject "relaxed and was cheerful after the session."

The data obtained allow us to outline the general dynamics of $H R V$ variables when the subjects effectively master the psychological relaxation methods during the teaching process: from CLS to ANS, from SNS to PNS, from the high/low total spectrum power to medium total spectrum power. These dynamics are very informative in terms of determining the degree of physiological lability of a subject. Very labile individuals are characterized by an extremely unstable state during the sessions. 
Deep immersion (the predominance of ANS and PNS) may give way to a dramatic increase in the SNS activity and a greater CLS influence. Such fluctuations are very characteristic of highly labile individuals during the first sessions.

\section{CONCLUSIONS}

(1) According to the results obtained, the predominance of the wave power of a very low frequency in the general spectrum (low $n L H$ values) may testify in favor of the leading activity of the corticolimbic brain structures in regulating the cardiac rhythm during psychological relaxation sessions. According to the psychological diagnosis data, such an increased activity is accompanied by neurotic symptoms or marked emotional excitation.

(2) The predominance of the autonomic nervous system in regulating the cardiac rhythm (high $n L H$ values) and parasympathetic influences in the autonomic balance (low $L F / H F$ values) is characteristic of deep immersion in the process of psychological relaxation sessions.

(3) The results of psychological diagnosis and spectral analysis allow us to suggest that asthenization processes are mediated by a significant decrease in the $H R V$ values (especially the total spectrum power $P W$ ) in the process of psychological relaxation. Thus, low $P W$ values may be indicative of reduction of the functional reserves of the organism.

(4) According to the data of psychological diagnosis, a combination of factors such as the autonomic level of cardiac rhythm regulation (high $n L H$ values), a low-level total spectrum power $(P W)$, and the predominance of the PNS in autonomic balance (low $L F / H F$ values) in the process of psychological relaxation sessions may indicate a predisposition to depression or the presence of a depressive state.

The results obtained require a more extensive and in-depth study. But it is evident that ///?V-analysis of the cardiac rhythm may serve as an effective physiological means of control and management of the process of psychological relaxation and diagnosis of a number of negative functional states in humans.

\section{REFERENCES}

1. Malik M. Heart rate variability. // Curr Opin Cardiol, 1998; 13(1): 36.

2. Task Force of the European Society of Cardiology and the North American Society of Pacing and Electrophysiology. Heart Rate Variability. Standarts of Measurements, Physiological Interpretation, and Clinical Use. //Circulation, 1996; 93: 1043.

3. Boulos M., Barron S., Nicolski E., Markiewicz W. Power spectral analysis of heart rate variability during upright tilt test: a comparison of patients with syncope and normal subjects. //Cardiology, 1996; 87:1, 28.

4. Mazerolles M., Senard J.M., Verwaerde P., Tran M.A.. Effects of pentobarbital and etomidate on plasma catecholamine levels and spectral analysis of blood pressure and heart rate in dogs. //Fundam Clin Pharmacol, 1996; 10:3, 298.

5. Panina G., Khot U.N., Nunziata E., Cody R.J., Binkley P.F. Role of spectral measures of heart rate variability as markers of disease progression in patients with chronic congestive heart failure not treated with angiotensin-converting enzyme inhibitors. // Am Heart J, 1996; 131:1, 153.

6. Liao D., Evans G.W., Chambless L.E., Barnes R.W., Sorlie P., Simpson R.J.Jr., Heiss G. Population-based study of heart rate variability and prevalent myocardial infarction. The Atherosclerosis Risk in Communities Study. //J Electrocardiol, 1996; 29:3, 189.

7. Liao D., Cai J., Barnes R.W., Tyroler H.A., Rautaharju P., Holme I., Heiss G. Association of cardiac autonomic function and the development of hypertension: the ARIC study. // Am J Hypertens, 1996; 9:12 Pt 1, 1147.

8. Кенделл М.Дж., Стьюарт А. Многомерный статистический анализ и временные ряды. - М.: Наука, 1976. - 736 с.

9. Баевский Р.М. Кибернетический анализ процессов управления сердечным ритмом. // Актуальные проблемы физиологии и патологии кровообращения. - М.: Медицина, 1976. - С.161.

10. Баевский Р.М. Прогнозирование состояний на грани нормы и патологии. - М.: Медицина, 1976. - 295 с.

11. Reardon M., Malik M. Changes in heart rate variability with age. //Pacing Clin Electrophysiol, 1996; 19:11 Pt 2, 1863.

12. Tsuji H., Venditti F.J., Manders E.S., Evans J.C., Larson M.G., Feldman C.L., Levy D. Determinants of heart rate variability. //J Am Coll Cardiol, 1996; 28:6, 1539.

13. Schultz S.K., Anderson E.A., van de Borne P. Heart rate variability before and after treatment with electroconvulsive therapy. //J Affect Disorders, 1997; 44:1, 13.

14. Evans D.L., Staab J., Ward H., Leserman J., Perkins D.O., Golden R.N., Petitto J.M. Depression in the medically ill: management considerations. //Depress Anxiety, 1996-97; 4:4, 199. 